Política y Sociedad

ISSN: $\quad 1130-8001$

ISSN-e: $1988-3129$

\title{
El papel de las mujeres en los acuerdos de paz en Colombia: la agenda internacional
}

\author{
Anabel Garrido Ortolá ${ }^{1}$
}

Recibido: 20-05.2018 / Aceptado: 17-02-2020

Resumen. Los acuerdos de paz en Colombia se han conformado como un referente internacional al poner fin a cincuenta y nueve años de conflicto armado en el país e incorporar nuevos instrumentos jurídicos que establece el marco sobre Mujer, Paz y Seguridad de Naciones Unidas. Así pues, esta transformación sobre las narrativas de la securitización está mediada por los relatos de las organizaciones de mujeres, que, junto con la Resolución 1325 del Consejo de Seguridad, han promovido su mayor participación en los procesos de negociación, así como la incorporación de un enfoque de género en los acuerdos de paz, logrando así romper con un espacio inicialmente masculinizado. A partir del examen de los distintos acuerdos de paz acontecidos en Colombia y las dinámicas de género que los atraviesan, el artículo evidencia cómo se ha logrado un cambio en las narrativas de género establecido en el discurso hegemónico, y cómo, en consecuencia, las mujeres se convierten en sujetos políticos necesarios para la consecución de la paz en Colombia.

Palabras clave: conflicto; procesos de paz; género; mujer; paz y seguridad.

\section{[en] Women's Role in Peace Agreements in Colombia: The International Agenda}

\begin{abstract}
Peace agreements in Colombia have become an international benchmark by bringing to an end fifty-nine years of armed conflicts in the country and assimilating new legal instruments in the United Nations framework on the Women, Peace and Security agenda. Thus, this transformation of securitisation narratives is mediated by the dialogues of women's organisations, which, in conjunction with Security Council Resolution 1325, have promoted their greater participation in negotiation processes, as well as the incorporation of a gender approach to peace agreements. Therefore, they have broken into an initially masculinized space. Based on the examination of the different peace agreements that have taken place in Colombia and the gender dynamics that permeate them, this article evinces how a change in gender narratives, based on hegemonic speeches, has been achieved and how, consequently, women have become political female-subjects necessary for achieving peace in Colombia.
\end{abstract}

Keywords: conflict; peace processes; gender; women; peace and security.

Cómo citar: Garrido Ortolá, A. (2020): "El papel de las mujeres en los acuerdos de paz en Colombia: la agenda internacional", Política y Sociedad, 57(1), pp. 77-97.

Universidad Complutense de Madrid (España).

E-mail: angarrid@ucm.es 
Sumario. 1. Introducción 2. La estratificación del género en la participación de la guerra. 3. Del objeto al método: las mujeres como sujetos políticos. 4. El papel de las mujeres como actores políticos en Colombia. 5. La inclusión de las mujeres en los acuerdos de paz y los programas de Desmovilización Desarme y Reintegración (DDR). 6. La agencia transformadora de las organizaciones de mujeres. 7. La agenda internacional como macronarrativa. 8. Conclusiones. 9. Bibliografía.

\section{Introducción}

Los acuerdos de paz realizados entre las FARC-EP y el Gobierno de Juan Manuel Santos pueden poner fin a cincuenta y nueve años de conflicto armado ${ }^{2}$ que ha fluctuado en diversas narrativas sobre la seguridad en el país.

Las políticas enmarcadas en la seguridad democrática impulsadas por Álvaro Uribe estuvieron caracterizadas por el cambio discursivo ${ }^{3}$ realizado después del 11 de septiembre. La criminalización de la insurgencia fue uno de los ejes principales. Se produce un cambio de denominación: de "guerrillas" a "grupos terroristas", y se realizó un vínculo con el narcotráfico: narcoterroristas o narcoguerrillas. Esta situación establecía un nuevo escenario de criminalización, no solo de la insurgencia, sino también de la ciudadanía organizada, donde todo aquel o aquella que no entendiese la "urgencia autoritaria por alcanzar la seguridad" sería un enemigo del sistema (Sader y Jinkings, 2009:342).

Con la llegada del Gobierno de Juan Manuel Santos, se produjo un cambio en las narrativas de seguridad, al generar un espacio de diálogo en 2012 donde se empezó a negociar la paz con una de las guerrillas más longevas de América Latina. Si bien es cierto que no son los primeros acuerdos de paz realizados hasta el momento en el país, sí son los primeros que pueden llevar hacia el fin de la insurgencia en Colombia.

Una de las referencias del proceso de paz en Colombia ha venido marcada por la incorporación de la voz de las mujeres en la agenda de seguridad y en la construcción de paz del país. Cabe preguntarse ¿cómo se ha incorporado la participación de las mujeres en el proceso de paz? y ¿cuáles son las narrativas que se desprenden de ellas? Para contestar a ambas preguntas, el artículo abordará la participación de las mujeres y las narrativas de género en la seguridad y la paz desde una aproximación sociopolítica. De este modo se abordará, por un lado,

2 Distintos hechos marcaron la conformación del conflicto armado en Colombia. En 1948 el asesinato de Jorge Eliecer Gaitán desencadenó el periodo denominado "La Violencia" (Wills, 2015:772). Posteriormente, la incorporación de El Frente Nacional como mecanismo político de alternancia en el poder (entre el Partido Liberal y el Partido Conservador) supuso una exclusión política de diversas sensibilidades en el país (Sader y Jinkings, 2009; Salamanca, 2008). Entre los años sesenta y setenta se conforman distintos grupos insurgentes de izquierda en el país, situación que desencadenó la inseguridad de las élites económicas, objeto de secuestro y extorsión (Gutiérrez, 2015). De este modo, en los años ochenta, en contraposición a la insurgencia se crearon grupos paramilitares, las denominadas Autodefensas Unidas de Colombia (AUC): grupos que se originaron de milicias rurales formadas por grandes terratenientes que tenían como fin llevar a cabo la lucha contrainsurgente. Durante los periodos gubernamentales posteriores, se realizaron acuerdos con distintas guerrillas en diversos momentos, y permanecieron activas hasta 2012 las Fuerzas Armadas Revolucionarias (FARC) y el Ejército de Liberación Nacional (ELN). El punto de referencia que se recoge para hacer el cálculo de 59 años de conflicto se retoma del surgimiento de las primeras guerrillas en 1960 .

3 El presente artículo no pretende realizar un análisis de discurso en torno a las narrativas internacionales, pero es necesario señalar que este cambio de discurso gubernamental viene determinado por un giro internacional neoliberal, acontecido después de 11 de septiembre, que llevará a la securitización de la agenda internacional (Buzan y Waever, 2009). 
cuáles son las narrativas de las organizaciones de mujeres como agencia transformadora; y por otro, cómo la agenda internacional ha ofrecido un marco para la inclusión de las mujeres en la construcción de paz en el país.

En esta línea, el punto dos establece un marco teórico que permite acercar el análisis de la participación sexuada en la construcción social de la guerra. Posteriormente, se aborda la metodología utilizada para el análisis, y en los dos puntos siguientes se revisa la participación de las mujeres en Colombia mediante los distintos acuerdos de paz y programas de Desarme Desmovilización y Reintegración (DDR) analizando: a) la participación de las mujeres como sujetos políticos, y b) la perspectiva de género en los mismos. En el punto sexto se abordan los relatos de las organizaciones de mujeres como eje transformador de las narrativas de género en el país; y en el séptimo, el componente internacional, la Resolución 1325 en la agenda de Mujer, Paz y Seguridad en Colombia. Por último, las conclusiones recogen algunas ideas señaladas.

\section{La estratificación de género en la participación de la guerra}

La guerra se evidencia como una construcción transcultural y atemporal donde uno de los géneros, el femenino, es excluido del campo de batalla. Esta participación sexuada $^{4}$ se debe a un desarrollo histórico de la guerra, que configura un orden social en torno a la polarización del género, junto con la expansión de la civilización y del poder del Estado (Malesevic, 2010). La estratificación de género no se encuentra referenciada en las organizaciones previas a las sedentarias, las organizaciones tribales basadas en el parentesco y en un liderazgo frágil sin la capacidad de un poder coercitivo (De Waal, 2005; Malesevic, 2010; Service, 1978). Así pues, será con el progreso de los "cacicazgos" y "estados prístinos" cuando el orden social se establecerá como jerárquico: se mostrará la estratificación de género en la configuración social y emergerán ambos hechos a la par (Malesevic, 2010:298).

Esta estratificación social se genera en torno a un cierre social que mediante elementos coercitivos y capitalistas, mantendría la consecución de las jerarquías en las cuales hubiera un ascenso de élites políticas (Mann, 1986; Tilly, 1985; Weber, 1964). En este sentido, los condicionamientos que llevan a la ausencia de las mujeres en los campos de batalla se derivan de los mecanismos de construcción en la organización social mediante la división sexual en dos esferas: la civil y la militar (Malesevic, 2010). Las mujeres han sido excluidas de la esfera militar, incluso aquellos hombres que no consiguen entrar son nombrados como mujeres; como señala Weber "quien no resiste las pruebas de guerra es una 'mujer', es decir, está excluido del séquito" (Weber, 1964:200). Esta división entre ambas esferas estructura una ideologización en torno a un discurso dicotómico, que muestra una jerarquía social donde la masculinidad subordina a la feminidad, es decir, a las mujeres, y a todo aquello definido desde "lo femenino".

Se habla de participación sexuada cuando el sexo biológico actúa como determinante en la participación, en este caso en la guerra. 
La representación de la masculinidad hegemónica ${ }^{5}$ y las posturas reificadas del género del sistema patriarcal ${ }^{6}$ vienen enunciadas por los discursos gubernamentales e institucionales, así como por la sociedad en su conjunto. Por tanto, este condicionamiento establecerá el escenario discursivo sobre la participación de las mujeres en la guerra como guerreras o sujetos activas de poder, que evidenciará la miopía con la que han sido atendidos tanto los procesos de desmovilización como los acuerdos de paz en Colombia.

La visión más extendida sobre el rol de las mujeres en las guerras es el de víctimas, vínculo que se instituye mediante la naturalización de víctima ligada al género femenino, al configurarse la identidad de género en torno al rol de mujeres como madres y guardianas de la cultura, y por tanto, como víctimas (Bennet et al., 1995). En este sentido, el análisis previo que diversos historiadores han realizado de la participación de las mujeres se centraba en torno a tres dimensiones: 1) como problema logístico; b) como dato estadístico; y c) como "sustitutas temporales" ante la ausencia masculina (Higonnet et al., 1987:43). Por tanto, la noción de la participación de las mujeres en la guerra se atendía desde una concepción pasiva; se las veía como población que proteger ${ }^{7}$.

La ceguera de género conforma parte del proceso de género (Cockburn, 1999), y es necesario el análisis del mismo para exponer "silencios inherentes a las actividades de mantenimiento de la paz" (Enloe, 2001:112). Esta posición neutral, al obviar la construcción de los roles de género, no solo no atiende al género femenino, sino que el género masculino se asume como neutro, hegemónico y reificado. La perspectiva de género no se integraba en los análisis sobre la violencia armada; la visión de la guerra era "un asunto de hombres" (Blair, 2012).

La lucha feminista ha conseguido, entre otras cosas, el impulso sobre los estudios de las mujeres, y ha generado la institucionalización de los mismos. Estos estudios han permitido criticar el carácter androcéntrico de los análisis sociales anteriores, que no tenían en cuenta la perspectiva de género. La proliferación de estudios sobre las dinámicas de género en la guerra ha mostrado que en los escenarios de conflicto armado, el sistema de dominación masculina se refuerza y acentúa (Cockburn, 2007; Magallón, 1998; Mesa, 2012). Además, se ha reconocido el impacto diferencial de género en los conflictos armados, visibilizando la violencia sexual en el cuerpo de las mujeres como una estrategia de guerra y no como un daño colateral. Las historias de vida sobre el relato de la violencia en sus cuerpos han sido fuente de informes, estudios y análisis. Asimismo, se han creado diversas organizaciones de mujeres que han generado resistencias hacia las narrativas de militarización social.

Del mismo modo, las organizaciones internacionales, haciéndose eco de estos estudios, han generado una agenda de Mujer, Paz y Seguridad en torno a la Resolución 1325 (2000), aprobada por el Consejo de Seguridad de Naciones Unidas, y reforzada por resoluciones posteriores. Esta resolución supuso un hito histórico al instar a los países a 1) la participación de las mujeres en todas las fases

El discurso hegemónico establece un orden androcéntrico y heterosexual que contempla distintas discriminaciones, entre las que se encuentra el sexo, pero también la etnia, clase social, identidad u orientación sexual, entre otras.

6 Las posturas reificadas del género del sistema patriarcal alude al discurso binario que estructura lo femenino y lo masculino.

Tratamiento que todavía persiste al aludir de forma conjunta a ancianos, niños y mujeres. 
de resolución de conflictos; 2) el enfoque de género que permita analizar la violencia diferencial que han sufrido las mujeres en la guerra. Así, la agenda de Mujer, Paz y Seguridad obliga a romper con la estratificación del género e interpela al concepto de seguridad internacional.

\section{Del objeto al método: las mujeres como sujetos políticos}

Ante el dominio masculino en la construcción ideológica del sujeto género, Spivak se preguntaba si el sujeto subalterno podría hablar. Y por tanto, si existe la posibilidad de que las mujeres pudieran conformar una narrativa de resistencia: "Si en el contexto de la producción colonial el individuo subalterno no tiene historia y no puede hablar, cuando ese individuo subalterno es una mujer su destino se encuentra todavía más profundamente a oscuras" (Spivak, 1998:20-21).

De este modo, Spivak alude a la falta de relato o historia de las mujeres como sujetos sociales en la configuración de narrativas propias. Esta visión negativa, ante la situación desigual en la construcción de narrativas propias, es rechazada al observar los movimientos de liberación. Como señala Edward Said, "en verdad, el subalterno puede hablar, como certifica la historia de los movimientos de liberación del siglo XX" (Said, 2002:440-441).

Al partir de la concepción de hegemonía narrativa, se atiende a la relación de poder entre una narrativa aceptada socialmente, frente a la marginalidad de relatos que se encuentran fuera de esta. Así, partiendo de la premisa de que donde existe poder existen "fenómenos de equivalencia y efectos frontera" (Laclau y Mouffé, 1987:231), la marginalidad deriva en la conformación de nuevos "contrapúblicos subalternos" que muestran visiones diversas a la hegemónica (Wills, 2007:65; Fraser, 1997:13).

En esta línea, para abordar la participación de las mujeres en el conflicto y los acuerdos de paz en Colombia, es necesario tener en cuenta las jerarquías de poder que establecen el posicionamiento del relato entre los actores, así como las resistencias y transformaciones de las narrativas hegemónicas.

La aportación que presento en el siguiente artículo deriva de un estudio más amplio que analiza las diversas narrativas de género en el conflicto armado y en los acuerdos de paz en Colombia ${ }^{8}$. Traída de esta investigación, en este estudio se abordan las narrativas hegemónicas y de resistencia que han configurado la inclusión o exclusión de las mujeres como sujetos políticos en la guerra y en la paz.

El artículo se aproxima al estudio desde una perspectiva sociopolítica, que toma en cuenta el análisis a través de los actores y las dinámicas de poder que estructuran esta participación. De esta manera, se analizan tres escenarios: las narrativas gubernamentales, las conformadas por las organizaciones de mujeres en Colombia y las derivadas de la agenda internacional.

Para el análisis de las narrativas gubernamentales, se estudian los diez acuerdos finales entre Gobiernos y guerrillas y AUC, el Pacto de Consolidación del Acuerdo Político entre el Gobierno nacional y la CRS (1996), el Informe de la Comisión Histórica del Conflicto y sus Víctimas (CHCV) de 2015 y el CONPES 2554/2008 de Delegación Nacional de Planeación (DNP).

Tesis doctoral defendida el 14 de diciembre de 2017. 
Las narrativas colectivas generadas por organizaciones de mujeres por la $\operatorname{paz}^{9} \mathrm{se}$ analizan mediante los documentos emitidos por estas organizaciones. La Organización Femenina Popular, a través de los medios de comunicación impresos: la revista anual de derechos humanos la Mohana (cuatro boletines "Voces de Mujeres") y el periódico bimensual Mujer Popular. La Ruta Pacífica, en los cincos documentos de "Agendas Mujeres por la Paz", trece boletines y varios documentos e informes. Y por último, la Red Nacional de las Mujeres mediante tres informes, los informes de la Coalición 1325 desde 2011 hasta 2016 y otras publicaciones.

Las narrativas internacionales se estudiarán como aquellas que se encuentran sobre las nacionales, y que configuran y condicionan las narrativas gubernamentales. En este caso, aquellas que se derivan de organizaciones internacionales, y que generan los relatos a través de la legislación, como la Resolución 1325 y posteriores, o mecanismos de concertación como las dos Cumbres Nacionales de Mujeres en Colombia.

\section{El papel de las mujeres como actores políticos en Colombia}

Las mujeres han ostentado distintos roles en el conflicto armado colombiano. Si bien son víctimas vivas de las distintas violencias acontecidas en el país, también han conformado distintos roles, tales como victimarias y sujetos políticos.

Según el Registro Unificado de Víctimas (RUV), las mujeres son poco más de la mitad de las víctimas: 4.132.427 frente a los 4.127.017 hombres víctimas (RUV, 2018). Al revisar el hecho victimizante, se puede observar un impacto diferencial de la violencia ante los "delitos contra la libertad y la integridad sexual", donde las mujeres víctimas de esta violencia son 22.363, en comparación con los 1.871 hombres (RUV, 2018).

No obstante, se debe de tener en cuenta que existe un alto subregistro en torno a la violencia sexual. Varias encuestas realizadas por distintas organizaciones así lo reafirman. En la encuesta de prevalencia, el 78\% de las mujeres víctimas de violencia sexual señalaba no haber denunciado; aproximadamente unas 620.418 mujeres (Sánchez et al., 2017). El 82,15\% de las mujeres entrevistadas por una investigación de Oxfam también enunciaba no haber denunciado frente a los hechos, y el 73,83\% indicaba la presencia de los grupos armados como obstáculos para la denuncia (Oxfam, 2010).

Aunque las mujeres son víctimas del conflicto armado, principalmente a través de la violencia sexual, el rol de víctimas es tan solo uno de los que refleja la participación de las mujeres en Colombia. La participación de las mujeres colombianas en las guerrillas, así como en las organizaciones de mujeres por la paz ha demostrado la falacia ante las categorías reificadas en torno al género, demostrando una "militancia activa como parte de su proyecto de vida y de sus actuaciones como sujetos políticos" (Herrera y Pertuz, 2014:151).

En las últimas décadas, el porcentaje de mujeres que se ha enrolado a los grupos armados ha aumentado de manera significativa en los grupos guerrilleros. En la década de los ochenta y noventa del siglo XX, entre el $24 \%$ y el $27 \%$ de las

Se analizará mediante el estudio de las narrativas de tres organizaciones: La Organización Femenina Popular (OFP), la Ruta Pacífica de las Mujeres y la Red Nacional de Mujeres (RNM). 
personas desmovilizadas en los acuerdos de paz eran mujeres. Uno de los porcentajes de mujeres más altos encontrados en la fecha refiere al M19, con un $31,5 \%$ de mujeres en su organización; y con menor proporción, la CRS $(17,5 \%)$. La participación de las mujeres en las FARC-EP se presume en torno al $40 \%$ (PNH, 1994; Turriago y Bustamante, 2003; Uricoechea, 2000).

Por tanto, la invisibilización de las mujeres en los espacios de poder contrasta con la realidad colombiana, donde la participación de las mujeres en el conflicto armado se encuentra en distintas esferas. No solo forman el $40 \%$ de los efectivos de las FARC-EP, sino que representan un papel importante como constructoras de paz en el país. Este es el caso de las organizaciones autónomas de mujeres por la paz en Colombia, que se han erigido como voces resistentes, y se han confrontado a la hegemonía narrativa. Esta confrontación deriva del propio sistema colombiano, que ha mostrado como "subversivo" todo aquello que se establece por fuera de los cauces gubernamentales e institucionales ${ }^{10}$ (Lamus, 2010; Wills, 2007), instituyendo así un marco de actuación polarizado, donde los efectos frontera marcan la posición contrahegemónica de las organizaciones de mujeres.

\section{La inclusión de las mujeres en los acuerdos de paz y los programas de Desmovilización Desarme y Reintegración (DDR)}

Las políticas en torno a la construcción de paz en Colombia han obviado la inclusión de las mujeres en los procesos de negociación. Este hecho se ha dado a través de dos vías: 1) mediante la participación de las mujeres en los espacios de participación y decisorios; 2) ante la ausencia de un enfoque de género que permita atender distintas realidades y fomentar una paz equitativa.

Para ello, es ineludible atender a "la conciencia de la diferencia". Esta conciencia ha de ir ligada con la construcción de los roles de género y cómo estos se instituyen en el patriarcado (Londoño y Nieto, 2006:85). Tan solo atendiendo a la diferenciación del sistema sexo-género, se podrán establecer los itinerarios pertinentes para la práctica investigadora que nos muestre las experiencias personales y colectivas en torno a las construcciones de género.

\subsection{La participación de las mujeres en los procesos de paz}

La participación de las mujeres colombianas en los procesos de paz se puede observar de diversas formas. Muchas mujeres han ocupado variados escenarios, que aun ocupando roles diferentes, han mostrado una gran capacidad para desempeñar las distintas funciones.

Por ejemplo, uno de los referentes como negociadora en Colombia fue Carmenza Cardona Londoño, conocida con su alias "la Chiqui" (M19). El papel desempeñado ante la toma de la embajada de la República Dominicana (1980) fue ejemplarizante, como referente del papel de las mujeres como negociadoras. No obstante, la participación de "la Chiqui" en la toma de la embajada de la República Dominicana estuvo condicionada por su género. Así pues, Rosemberg Pabón

10 Aunque en este caso refiere a la participación de las organizaciones de mujeres en la política estatal de Colombia, esta polarización no se da únicamente con ellas, sino con todas las organizaciones de derechos humanos o de víctimas, criminalizándoles al establecer una similitud entre la insurgencia y estas. 
Pabón, conocido como el Comandante Uno, señala la discriminación por parte de los representantes del Gobierno al tener que negociar con una mujer:

Lo que más les dolía a los representantes del Gobierno era hablar con una mujer encapuchada, gordita, bajita. "Pero usted... ¿puede decidir?", decían como burlándose. Esperaban al Comandante Uno (Pabón, 1984:71-72).

Aunque el papel de "la Chiqui" se ha mostrado como un referente, y forma parte del imaginario colectivo en la sociedad colombiana, se evidencia como un hecho aislado. Los posteriores acuerdos de paz muestran un mapa caracterizado por la ausencia de participación de las mujeres en los espacios gubernamentales e institucionales decisorios (Velásquez, 2009). Al recoger la participación de las mujeres en los distintos acuerdos de paz entre el Gobierno de Belisario Betancur (1982-1986) y los grupos guerrilleros, tan solo 4 mujeres $(7,14 \%)$ aparecen en los cuatro acuerdos realizados (Chaparro y Martínez, 2016).

Posteriormente, en el Gobierno de Andrés Pastrana, de los 18 acuerdos realizados aparecerían 149 hombres y 3 mujeres (1,97\%). Sin embargo sería todavía mucho más evidente la falta de participación de las mujeres en el periodo de Álvaro Uribe (2002-2010), donde ante la firma de 12 acuerdos negociados con 64 hombres, no habría ninguna mujer (Chaparro y Martínez, 2016) (tabla 1). Según la revisión ${ }^{11}$ sometida por Londoño y Nieto, solo 15 mujeres de 280 hombres aparecen como signatarias en los acuerdos (Londoño y Nieto, 2006).

Esta realidad muestra que las mujeres - como signatarias gubernamentales y/o guerrilleras - no aparecen en las mesas de negociación o en aquellos espacios como foros y consejos institucionales donde se negocia la paz (tabla 1).

Tabla 1. Presencia de hombres y mujeres en las mesas de negociación (\% de participación)

\begin{tabular}{|l|c|c|}
\hline & Hombres (\%) & Mujeres (\%) \\
\hline Belisario Betancur & 92,86 & 7,14 \\
\hline Andrés Pastrana & 98,03 & 1,97 \\
\hline Álvaro Uribe & 100 & 0 \\
\hline Juan Manuel Santos & 84,31 & 15,69 \\
\hline
\end{tabular}

Fuente: Tomado de Chaparro y Martínez (2016:79).

Aunque el inicio del proceso de paz con el Gobierno de Juan Manuel Santos y las FARC-EP se caracterizaría por la falta de inclusión de mujeres en el mismo, gracias a la reivindicación de las organizaciones de mujeres, la participación de ellas como negociadoras se incrementó a 43 hombres y 8 mujeres: un 15,69\% (Chaparro y Martínez, 2016).

1 La revisión se realiza por medio de "veinticinco acuerdos, dos declaraciones conjuntas, un informe de comisión, un acta de comisión y una agenda (incluidos los [...] acuerdos con los grupos paramilitares en Santa Fe de Ralito)" (Londoño y Nieto, 2006:62). 
No obstante, al abordar la participación de las mujeres desde el trabajo de apoyo en distintas comisiones, podemos observar no solo el incremento en el porcentaje de participación femenina con respecto a la participación de las mujeres como signatarias, sino también que en algunos casos el porcentaje de mujeres es mayor al de hombres. En esta línea, podemos observar un porcentaje del $62,92 \%$ de participación femenina con el Gobierno de Andrés Pastrana, y un 65,78\% con el Gobierno de Juan Manuel Santos (tabla 2).

Tabla 2. Presencia de hombres y mujeres en las comisiones de apoyo a las negociaciones (\% de participación)

\begin{tabular}{|l|c|c|}
\hline & Hombres (\%) & Mujeres (\%) \\
\hline Belisario Betancur & 92,29 & 9,71 \\
\hline Andrés Pastrana & 37,08 & 62,92 \\
\hline Álvaro Uribe & 61,54 & 38,46 \\
\hline Juan Manuel Santos & 34,22 & 65,78 \\
\hline
\end{tabular}

Fuente: tomado de Chaparro y Martínez (2016:79).

Otro ejemplo de la participación sesgada de las mujeres se observa en la redacción del Informe de la Comisión Histórica del Conflicto y sus Víctimas (CHCV), donde se evidencia el poco reconocimiento hacia las mujeres colombianas pertenecientes a la academia. El informe fue realizado por 14 personas académicas, 12 articulistas y 2 relatores elegidas entre el Gobierno y las FARC-EP, de los cuales tan solo una era una mujer, la profesora María Emma Wills. Como se cuestiona Rodrigo Uprimny, “¿no había acaso más académicas dignas de integrar esta comisión?" (Uprimny, 2014). Ante esta pregunta se responde enumerando distintas mujeres académicas que han trabajo sobre el conflicto armado colombiano, y finaliza con una pregunta retórica que muestra por qué se da esta realidad: “¿O será que ambos ven la guerra como un asunto puramente viril, por lo cual creen que su documentación e interpretación es una historia de solo hombres?" (Uprimny, 2014).

Además, al margen de la escasa participación femenina en esta comisión, la atención sobre las dinámicas sexuadas de la guerra es casi inexistente. Debido a ello, varias organizaciones feministas y de mujeres enviaron una comunicación al considerar que "si bien en algunos de los documentos se hace mención a la situación de las mujeres y se nombran algunos de los delitos de los que fueron víctimas, ninguno de ellos recogió la complejidad de lo vivido por las mujeres" (Ramírez, 2015:71).

Estas dinámicas patriarcales forman parte de todos los procesos, y debido en parte por ello, la subcomisión de género incorporó en el segundo punto del acuerdo final la necesidad de promover la participación de las mujeres. De este modo, en torno a la "Participación política: Apertura democrática para construir la paz" (Mesa de conversaciones, agosto 2016:30), se indica la necesidad de la 
participación de las mujeres debido a que "ellas enfrentan mayores barreras sociales e institucionales para el ejercicio de su participación política como consecuencia de profundas discriminaciones y desigualdades" (Mesa de conversaciones, agosto 2016:30). Por consiguiente, se establece el compromiso de promover, garantizar y no estigmatizar a las mujeres como sujetos políticos, al igual que se insta al Gobierno y las instituciones al fortalecimiento de las organizaciones de mujeres (también se señalan las organizaciones de jóvenes y de población LGTBI). En el punto 2.3.7 se indica la promoción de la participación de las mujeres en el presente acuerdo: "El Gobierno Nacional y las FARC-EP reconocen el importante papel que desempeñan las mujeres en la prevención y solución de los conflictos y en la consolidación de la paz, y la necesidad de promover y fortalecer la participación política y ciudadana de las mujeres" (Mesa de conversaciones, agosto 2016:48).

\subsection{La ausencia de la perspectiva de género}

Los procesos de desmovilización en Colombia han estado caracterizados por la denominada "posición neutral frente al género" (Londoño y Nieto, 2006) ${ }^{12}$. Esta denominación permite vislumbrar un posicionamiento: la homologación al género masculino, lo que refuerza la visión patriarcal al invisibilizar el género femenino en la posición de victimario. Como afirma María Eugenia Vásquez, "la homologación desde lo masculino mantiene vigentes relaciones de dominación de la sociedad que no se transforman mecánicamente, porque están arraigadas en estructuras mentales y concepciones mucho más profundas que las esbozadas en una propuesta de cambio político a secas" (Vásquez, 2001:7).

En los contenidos de los acuerdos ${ }^{13}$ no se han encontrado la mención a las mujeres, a la integración de un enfoque de género o a reivindicaciones en torno a los derechos de las mujeres desmovilizadas. La única mención hacia las mujeres se encuentra recogido en el "Programa Integral de Viudas y Huérfanos de miembros de la CRS" para las viudas de los combatientes en el marco de un acuerdo entre el Gobierno y la CRS (Pacto de Consolidación del Acuerdo Político entre el Gobierno nacional y la CRS, 1996; Londoño y Nieto, 2006:63). Esta carencia de perspectiva de género vendría determinada porque "siempre se miró el mundo de la militancia y de la reinserción en neutro, es decir en masculino" (Colectivo de Mujeres Excombatientes, 2001).

De este modo, como se corrobora en la siguiente entrevista a un dirigente de la CRS:

12 El trabajo realizado por las profesoras María Luz Londoño y Yoana Nieto, "Mujeres no contadas", es uno de los más completos sobre los programas de DDR en Colombia. Debido a ello, y al poco material encontrado sobre el tema, es una de las referencias más señaladas en este epígrafe.

13 Se ha hecho una revisión entre los distintos acuerdos realizados con el Gobierno y las guerrillas en la década de los ochenta y noventa: acuerdo final entre el Gobierno nacional y el grupo armado Quintín Lame; acuerdo político entre el Gobierno nacional, los partidos políticos, el M19, y la iglesia católica en calidad de tutora moral y espiritual del proceso; acuerdo final entre el Gobierno nacional y el EPL; acuerdo político final entre el Gobierno nacional y la CRS; acuerdo final entre el Gobierno nacional y el PRT; acuerdo entre el Gobierno nacional y los comandos Ernesto Rojas; acuerdo final entre el Gobierno nacional y las Milicias Populares de Medellín; acuerdo final entre el Gobierno nacional y la ADO, y acuerdo final entre el Gobierno nacional y el Frente Francisco Garnica de la Coordinadora Guerrillera. 
(...) en los acuerdos tampoco existe la mujer y el tema de la mujer. En los de la Corriente creo que, sin ningún ánimo vanguardista ni nada por el estilo, empezó a aparecer muy tímidamente. Incluso no aparece en el pacto de 1994, sino que aparece en un documento que se llamó «El Pactó de Consolidación de los Acuerdo de Paz», que se firmó en 1996 con el Gobierno de Samper, en donde apareció el «Programa de atención integral a viudas y huérfanos». En ese momento, transcurridos casi dos años desde la desmovilización, nos dimos cuenta de que hay unos problemas muy específicos de las mujeres — no estamos todavía abordando el tema de género, la perspectiva de género- que requieren políticas diferenciales y ofertas específicas, que no existían y se perdían en la generalidad del tema de la reinserción. (...) en el caso de la Corriente nosotros lo entendimos ya en el terreno propio de la reinserción, cuando empezamos a enfrentarnos a situaciones ya no solo de lisiados de guerra sino de compañeras que están viudas, solas, afrontando no solo la condición de reinsertada sino afrontando solas la crianza de los hijos, afrontando el dolor, el duelo de la muerte, con hijos también afectados por situaciones dramáticas de la guerra que exigían un tratamiento particular muy distinto a cualquier otro desmovilizado (Londoño y Nieto, 2006: 63-64).

Este hecho es significativo, ya que, de todos los acuerdos realizados en la década de los ochenta y noventa, esta es la única alusión a las mujeres, y se realiza bajo los términos de las viudas de los excombatientes. Este programa, desarrollado por la Corporación Nuevo Arco Iris entre 1997 y 1998, se enfocó en "actividades de formación, de encuentro y asistencia psicosocial", además de un bono de apoyo escolar y 76 créditos para iniciativas empresariales de las mujeres viudas (Corporación Nuevo Arco Iris, 2005:14). La alusión única a mujeres como "las mujeres de" marca la hegemonía narrativa en la que, por un lado, no se atiende a la situación específica de las mujeres desmovilizadas; y por otro, esta discriminación sexuada actúa negando a la mujer desde otro rol que se le asigna en la norma. Para ello, es necesario partir de "la conciencia de la diferencia". Esta conciencia ha de ir ligada con la construcción de los roles de género, y cómo estos son construidos desde el sistema de dominación masculina.

A partir de julio de 1992, el Programa de Reinserción implementado por el Estado empezó a tomar en consideración el alto porcentaje de mujeres desmovilizadas, sin embargo, en ningún caso el tema de la mujer se incluyó en los espacios de evaluación de políticas y programas de DDR gubernamentales (Londoño y Nieto, 2006). En el Foro sobre Aspectos Institucionales de la Reinserción no se contó con ninguna mujer desmovilizada, pero se realizaron 24 entrevistas a hombres reinsertados (Murillo, 2004:89). El Informe Nacional de Desarrollo Humano de 2003 tampoco reconoce a las mujeres como sujetos del proceso, de esta manera en el capítulo diez, titulado "Deshacer los ejércitos: la desvinculación de combatientes", se opta por un lenguaje masculino, una vez más integrando el enfoque "neutro", y muestra el género femenino únicamente cuando se alude a las víctimas y a menores, como en "los niños y las niñas" (PNUD, 2003:233).

Si bien los procesos de paz acontecidos en las décadas anteriores no contaron con un enfoque de género y con una participación reducida de mujeres, en los acuerdos con el paramilitarismo el enfoque de género fue igualmente inexistente, $y$ 
la participación de las mujeres fue incluso menor que en la década de los ochenta y noventa (CNRR, 2010). Por consiguiente, en los acuerdos de Punto Final entre el Gobierno y las AUC (2004-2005), no solo no se incorporó una análisis de género sobre las diversas realidades entre desmovilizados y desmovilizadas, sino que no se introdujeron medidas específicas que atendieran a las mujeres, e incluso contó con una participación menor que en los anteriores procesos (CNRR, 2010).

Entre la exclusión de las mujeres de los procesos de paz en el país, podemos señalar tres ejes que marcan esta situación:

\section{— La exclusión de las mujeres en las cifras}

El desconocimiento de las personas desmovilizadas ha sido uno de los principales problemas, debido a la falencia de registro en los procesos colectivos de desmovilización, la dispersión de archivos y los cambios de entidades responsables de los programas. Este hecho se agrava en el caso de las mujeres, al no contar con un sistema desagregado de datos por sexo (Londoño y Nieto, 2006).

\section{— La exclusión de las mujeres en la palabra y en la ley ${ }^{14}$}

Este apartado refiere al empleo exclusivo del término masculino para referir a las personas desmovilizadas en el conflicto armado colombiano. De este modo, se evidencia la integración de lo que se denominaba anteriormente como el "enfoque neutro" o la "homologación de lo masculino". El uso del lenguaje utilizado en los acuerdos ha nombrado tan solo desde lo masculino: "desmovilizados", "guerrilleros", "reinsertados", sin aludir en ningún caso a las mujeres partícipes de los grupos armados ilegales. Igualmente, la ley colombiana no hace mención diferenciada sobre el género, por lo que vuelve al denominado "enfoque neutro", que no deja de ser sino la homologación masculina de la ley.

\section{— La reificación de las mujeres en torno a la familia}

La atención que se desprende de las mujeres excombatientes en los documentos políticos sobre la desmovilización refiere al vínculo de ellas dentro de la familia. Si bien del acuerdo con la CRS se desprende la atención de las mujeres viudas de excombatientes de la CRS, en el documento de Conpes 3554/2008 del DNP se especifica: "La desmovilizada que toma la decisión de dejar las armas y entregarse voluntariamente asume el compromiso de construir y promover el crecimiento de su familia; y en el caso de ser la pareja del desmovilizado, adicionalmente su rol se orienta a motivar la permanencia de su compañero en el proceso. Las medidas que se apliquen, con el apoyo técnico de la Consejería Presidencial para la Equidad de la Mujer, estarán dirigidas a fortalecer estos roles" (CONPES 3554/2008, DNP).

Así, se deduce que la mujer ha de actuar como garante familiar, ya bien sea mediante su compromiso como desmovilizada en la construcción y promoción de su propia familia, o bien como compañera sentimental del desmovilizado, entendiendo que en ambos casos el papel de la mujer ha de ser el motor familiar.

Análisis a través de los diez acuerdos finales entre los Gobiernos y grupos irregulares, el Pacto de Consolidación del Acuerdo Político entre el Gobierno nacional y la CRS (1996), y el Informe de la Comisión Histórica del Conflicto y sus Víctimas (CHCV) de 2015. 
Del mismo modo, se señala como función de la Consejería Presidencia para la Equidad de la Mujer el establecer medidas que sirvan para el fortalecimiento de estos roles normativos.

Este silencio establecido por el Gobierno y las instituciones, a través de sus documentos políticos y leyes, invisibiliza la participación de las mujeres como victimarias, y cuando se identifica la participación activa de ellas, se referencia la familia como eje vertebrador de su condición de género. La invisibilización de las mujeres como excombatientes debido a la falta de atención diferencial en el proceso de la desmovilización retrae a las concepciones previamente construidas por el sistema de dominación masculina.

El discurso hegemónico binario en torno a la construcción de los roles se reifica en el discurso gubernamental e institucional al concebir únicamente los procesos de desmovilizaciones, ya sean dentro de unos acuerdos de paz o dentro de los procesos individuales, como un proceso únicamente masculino. Desde los acuerdos de paz de los ochenta y noventa hasta los procesos de DDR impulsados por el Gobierno de Álvaro Uribe, el proceso de desmovilización y reintegración se ha caracterizado por la carencia de un enfoque de género. La Comisión Nacional de Reparación y Reintegración señala las carencias del enfoque de género, así como de la atención diferenciada de la agenda de mujeres desmovilizadas: "En todos los acuerdos de paz y en los procesos de DDR aplicados en Colombia, (...) se carece de un enfoque de género, se deja de lado a las excombatientes, se desconocen sus agendas, intereses y necesidades, y se desestima u obstaculiza su acceso a los beneficios previstos" (CNRR, 2010:220).

\section{La agencia transformadora de las organizaciones de mujeres}

La participación de las mujeres colombianas en organizaciones sociales ha conformado grupos de "resocialización", donde la identidad previa se resignifica a través de los relatos compartidos (Gorlier, 2002). De este modo, las narrativas colectivas establecidas en las organizaciones conforman nuevos roles "y en las colectividades con más roles que miembros, estos tienen la oportunidad no solo de ser ayudados, sino también de ayudar" (Gorlier, 2002:38). En esta línea, la Ruta Pacífica de las Mujeres utiliza el concepto de "sobrevivientes" para aludir a la situación de víctima, resignificando la concepción de víctima mediante el uso de un concepto del cual se desprende la noción de sujeto activo y, por tanto, de empoderamiento.

El acercamiento de muchas mujeres a estas organizaciones ha condicionado nuevos escenarios, ya que, independientemente del motivo del acercamiento a la organización, la participación activa en la lucha política ha promovido la generación de narrativas compartidas. Según el Informe de la Verdad de las Mujeres de la Ruta Pacífica, en torno al $57 \%$ de las mujeres entrevistadas señalaban una transformación de su identidad, al asumir roles que la sociedad colombiana no vincula con las mujeres (Ruta Pacífica de las Mujeres, 2013). Este cambio de rol de víctima a sujeta política les hace ganar agencia mediante distintas vías: "Las mujeres afirman sentirse diferentes después de estas experiencias que las cambiaron fortaleciéndolas, dándoles mayor humanidad, autoconocimiento, 
independencia y autoestima. Señalan que en la relación con las parejas y los hijos cambiaron, haciéndose respetar más y aprendiendo a resolver conflictos sin violencia. Ganaron en su capacidad de defenderse pero también en su capacidad de ayudar a las demás personas" (Ruta Pacífica de las Mujeres, 2013:83).

Por lo tanto, la conformación de sujetos políticos genera un empoderamiento individual, así como grupal, lo que permite una mayor agencia y amplitud de las capacidades personales (Squires, 2000). No obstante, este empoderamiento ha generado situaciones de peligro para las mujeres. La participación activa de las mujeres como sujetos políticos ha estado condicionada por la situación de víctimas, debido a una situación previa a la participación o por motivo de ella. Asimismo, muchas mujeres lideresas han sido amenazadas, y han tenido que optar por bajar su perfil político o "disminuir su visibilidad" para evitar la violencia sobre ellas (Ruta Pacífica de las Mujeres, 2013:84). En algunos casos se observa la necesidad de ocultar el empoderamiento conseguido, debido a la violencia aleccionadora que establece la hegemonía narrativa por no acatar los mandatos del género.

Al margen de las vicisitudes derivadas del empoderamiento, la amplitud de agencia se erige como uno de los elementos cruciales en las organizaciones de mujeres. Mediante el proceso de duelo, anclajes y movilización, las mujeres víctimas evidencian una transformación identitaria al distanciarse de los roles y relatos pasados (White, 1992), conformando una nueva identidad grupal en torno a las narrativas colectivas (Gorlier, 2002).

La piedra angular del discurso en las organizaciones de mujeres deriva de su condición de mujer, por tanto, el cuerpo se convierte en sujeto político de reivindicación. Parte de las demandas se realizan a través de la denuncia del impacto diferencial que la guerra genera en el cuerpo de las mujeres. Sin embargo, si bien el cuerpo se instituye como elemento por el cual opera la dominación —-la violencia sexual como objetivo de guerra-, también se muestra como el lugar donde se genera la resistencia. El cuerpo se erige como sujeto político por el cual media el relato y las estrategias de las organizaciones de mujeres. En esta línea, se pueden señalar como acciones de resistencia la ocupación de espacios masculinizados en dos líneas diferenciadas:

1) La ocupación de la calle, entendida como espacio de lucha propio del cuerpo (Butler, 1993). En este caso tanto la Ruta como la OFP optarán por esta vía de acción colectiva a través de manifestaciones, plantones y caravanas. La calle plantea una reivindicación directa con el espacio público masculinizado, y genera identificación y encuentro con otras mujeres.

Igualmente, estas acciones se llevan a cabo a través de una simbología propia de las mujeres, "símbolos construidos a partir de experiencias, deseos y formas de estar en el mundo: vehiculadas todas ellas a través de teatros, rituales, fiestas y cantos en donde el cuerpo - los cuerpo pintados, principalmente - centren el eje de lucha" (Sánchez y Rodríguez, 2015:166).

2) La ocupación de espacios gubernamentales e institucionales de participación política. Estos espacios igualmente masculinizados son los espacios de 
reivindicación e incidencia principales en la $\mathrm{RNM}^{15}$. De este modo, la reivindicación se plantea a través de los derechos humanos de las mujeres, situando el foco en la necesidad de la participación de estas en los organismos e instituciones que lo requieran.

La ocupación de las calles por parte de la Ruta genera nuevas dinámicas de resistencia, ya que buscan reapropiar[se] de lo simbólico y así romper con las dinámicas masculinas que impone la guerra (Sánchez, y Rodríguez, 2015:166). Mediante el baile y el color en la calle pretenden romper con los códigos construidos del sistema de dominación masculina (Millet, 1975). Una de las razones por la cual se observa esta ruptura empieza cuando estas se convierten en una "amenaza al discurso homogeneizante y hegemonizante de la guerra" provocando "malestar en los señores de la guerra" (Camilo, 2006:13).

Los objetivos de la Ruta son tanto la construcción de paz en el país como el reclamo hacia la ruptura del sistema de dominación, al plantear una nueva negociación de las relaciones entre hombres y mujeres (Cifuentes, 2009). En los relatos de la organización, se establecen cuestionamientos constantes hacia el poder, la guerra y la política del sistema sexo-género (Sánchez, 2006).

Las diversas realidades han configurado distintas narrativas que en torno a la construcción de paz y equidad de género se establecen como resistencias ante el sistema de dominación masculina. El "poder para" se genera en procesos individuales y colectivos, concibiendo una mayor agencia. Distintos han sido los logros de las organizaciones de mujeres por la paz en el mundo. Entre ellos se encuentra la ruptura entre la concepción privada versus pública de la violencia de género, la sororidad entre mujeres y la conformación de espacios de reivindicación.

Varios son los resultados que las organizaciones de mujeres en Colombia han conseguido a través de las movilizaciones, programas e incidencia política; desde el acompañamiento y la sororidad entre mujeres víctimas hasta la transformación identitaria de víctimas a sujetos políticos. Uno de los mayores logros conseguidos en Colombia, que se conformó como un hito internacional, es la consecución del enfoque de género y una mayor participación femenina en los acuerdos de paz entre las FARC-EP y el Gobierno de Juan Manuel Santos. Gracias al movimiento social de mujeres, que revirtió el proceso masculino, se consiguió incidir en el incremento de la participación femenina, y la generación de un espacio técnico que promoviera la incorporación de una perspectiva de género en todo el proceso de paz.

\section{La agenda internacional como macronarrativa}

No obstante, la participación de las mujeres y la incorporación de la perspectiva de género en los acuerdos de paz en Colombia no hubieran sido posibles sin el marco normativo internacional que insta a los Gobiernos a generar una agenda de seguridad, paz y género.

Esto no quiere decir que la Ruta o la OFP no realicen acciones de cabildeo en los partidos políticos, o que la RNM no realice movilizaciones ocupando el espacio público. Tan solo muestra cuáles son los espacios de presión donde se erigen principalmente sus reivindicaciones. 
En 2012 el inicio del proceso de diálogo con las FARC-EP se caracterizó por la ausencia de mujeres en las mesas de negociación. La exclusión inicial de las mujeres en los diálogos de paz genera procesos paralelos, donde las organizaciones de mujeres reivindican sus demandas, y en algunas ocasiones se llegó a acuerdos por esta vía (ONU Mujeres, 2011). La organización de estos procesos es uno de los métodos más empleados por parte de ellas, y se genera mediante la reacción ante la exclusión en los procesos formales.

En Colombia esta referencia se encuentra en las dos Cumbres Nacionales de Mujeres realizadas en 2013 y 2016. La I Cumbre Nacional de Mujeres (2013), apoyada por la comunidad internacional bajo los lemas "Sin mujeres no hay paz" y "Las mujeres queremos ser pactantes y no pactadas", que invocaron a la participación equitativa de las mujeres en los acuerdos de paz, fue el motor que revirtió el inicio masculinizado de los diálogos.

Estas cumbres se ofrecieron apoyo económico y técnico, y por ende político, por parte del Sistema de las Naciones Unidas en Colombia, liderado por ONU Mujeres, y con el apoyo de la cooperación de Suecia, Noruega, Suiza y España (I Cumbre Nacional de Mujeres y Paz, 2013). Estas reivindicaciones condicionaron el incremento de la participación (con el nombramiento de dos mujeres como plenipotenciarias) y la creación de la subcomisión de género integrada en el proceso de paz. La subcomisión se ha erigido como un espacio con un importante componente transformador que ha permitido la incorporación del enfoque de género en todos los puntos del acuerdo de paz entre las FARC-EP y el Gobierno nacional.

Así pues, los compromisos internacionales en la Resolución 1325 han influido en las narrativas gubernamentales en Colombia, a través del incremento de la participación de las mujeres y la incorporación del enfoque de género integrado en el acuerdo final entre las FARC-EP y el Gobierno. El documento está redactado en un lenguaje no sexista, y la aparición de la palabra "mujer" se contabiliza en 197 veces (Mesa de conversaciones, agosto 2016). Este acuerdo supone un hecho histórico, ya que por primera vez se acerca al cumplimiento de la Resolución 1325.

\section{Conclusiones}

La guerra y la construcción de esta en torno al sistema de dominación masculina nos muestra un mundo de hombres, donde se invisibiliza a las mujeres que forman parte de los grupos armados, así como la participación de las mujeres como sujetos políticos. Pareciera que el único espacio en el que se acepta la participación de las mujeres es aquel que les corresponde en la guerra en función de su "condición" de género, el de víctimas. Al retomar la pregunta inicial ¿cómo se ha incorporado la participación de las mujeres en el proceso de paz y cuáles son las narrativas que se desprenden de ellas?, podemos observar que ha habido una evolución en Colombia.

Los procesos de desmovilización en Colombia realizados en las décadas anteriores han estado caracterizados por la denominada "posición neutral frente al género". Esta denominación permite vislumbrar un posicionamiento claro, el que se homologa al género masculino. La invisibilización de las mujeres de los espacios de poder contrasta con la realidad colombiana, donde la participación de 
las mujeres en el conflicto armado se encuentra en distintas esferas, forman parte de los efectivos de las guerrillas y son sujetos políticos en la construcción de paz en el país.

Sin embargo, uno de los principales hitos en el proceso de paz con las FARC-EP ha estado relacionado con el incremento de la participación de las mujeres y la incorporación de nuevos mecanismos de concertación en las negociaciones de paz: la creación de la subcomisión de género como espacio técnico para la introducción de una perspectiva de género en los acuerdos de paz. La creación de la subcomisión de género no hubiera sido posible sin la comunidad internacional, a través del apoyo económico y político de Naciones Unidas y varias embajadas. Igualmente, hubiera sido impensable sin el papel de las mujeres de la sociedad civil organizada en Colombia, que mediante la incidencia — ante la ausencia de mujeres en las negociaciones iniciales-, consiguieron incorporar sus demandas en el proceso de paz. Asimismo, la Cumbre Nacional de Mujeres (2013) se constituyó como un espacio de debate y construcción entre las organizaciones de mujeres, que instó al Gobierno hacia el deber, no solo de la participación de las mujeres en el proceso, sino de la incorporación de una perspectiva de género, cumpliendo con los compromisos ratificados mediante la Resolución 1325.

En consecuencia, la narrativa hegemónica en Colombia —enunciada principalmente por el Gobierno y las instituciones - ha ignorado de los procesos de guerra y paz a las mujeres como sujetos políticos, y también las ha ignorado como actores armados (mujeres excombatientes) en los procesos de desmovilización. Al establecer una mirada global sobre los distintos procesos acontecidos en Colombia, en un promedio general, desde los acuerdos de Belisario Betancur hasta los actuales con Juan Manuel Santos, la participación de las mujeres en los procesos de paz ha sido de un 4,07\% .

Los acuerdos realizados con las FARC-EP, aun contando con una mayor participación femenina, siguen lejos de tener una participación equitativa. Empero, la creación de instrumentos como la subcomisión de género se erige como uno de los espacios con un mayor componente transformador, que ha promovido la participación de mujeres como sujetos políticos (guerrilleras y responsables gubernamentales), lo que ha conseguido transversalizar el género en los distintos puntos acordados entre el Gobierno y las FARC-EP.

Estos logros se han conseguido por la agencia transformadora de las organizaciones de mujeres en el país, y por el apoyo de la comunidad internacional bajo el paraguas de la Resolución 1325. Porque la seguridad internacional tiene que llegar a las mujeres, porque tan solo con ellas la construcción de paz será posible en Colombia.

\section{Bibliografía}

Bennet, O., J. Bexley y K. Warnock (eds.) (1995): Arms to fight, arms to protect: women speak out about conflict, Londres, Panos.

Blair, E. (2012): Un itinerario de investigación sobre la violencia. Contribución a una sociología de la ciencia, Colombia, Universidad de Antioquia.

Butler, J. (1993): Cuerpos que importan, Buenos Aire, Paidós. 
Buzan, B. y O. Wæver (2009): "Macrosecuritisation and security constellations: reconsidering scale in securitisation theory", Review of International Studies, 35, pp. 253-276.

Camilo, A. L. (2006): Las mujeres paz haremos movilizándonos contra la guerra. Actoras politicas, desobedientes civiles, Minneapolis, Centro para Víctimas de la Tortura.

Chaparro, N. y M. Martínez (2016): "Negociando desde los márgenes. La participación de las mujeres en los procesos de paz en Colombia 1982-2016”, Ideas para construir la paz, Documentos 29, Bogotá, Dejusticia.

Cifuentes, M. R. (2009): "La investigación sobre género y conflicto armado", Eleuthera, Vol. 3, pp. 127-164.

CNRR (2010): La reintegración logros en medio de rearmes y dificultades no resueltas. II Informe de la Comisión Nacional de Reparación y Reconciliación, Bogotá, Comisión Nacional de Reparación y Reconciliación.

Cockburn, C. (1999): “Género, conflicto armado y violencia política”, en Cuadernos INER, Washington D.C, Banco Mundial.

Cockburn, C. (2001): "The Gendered Dynamics of Armed Conflict and Political Violence", en C. N. O. Moser y F. Clark (eds), Victims, Perpetrators or Actors?: Gender, Armed Conflict and Political Violence, Londres, Zed Books.

Cockburn, C. (2007): Mujeres ante la guerra, Barcelona, Icaria.

CONPES 3554/2008, DNP, 1 de diciembre, política nacional de reintegración de excombatientes.

Corporación Nuevo Arco Iris (2005): Diez años después... Un balance del proceso de paz, Bogotá, Corporación Nuevo Arco Iris. Disponible en:

http://www.cedema.org/uploads/CRS(10).pdf, último acceso, 06 de septiembre de 2017. [Consulta: 28 de abril de 2018]

Cumbre Nacional de Mujeres y Paz (octubre 2013): "I Cumbre Nacional de Mujeres y Paz", Bogotá, Colombia, octubre 23 al 25 de 2013, Sistematización de la I Cumbre Nacional de Mujeres y Paz”, Bogotá.

II Cumbre Nacional de Mujeres y Paz (septiembre 2016): Manifiesto político "Las mujeres vamos a por la paz”, Bogotá.

De Waal, F. (2005): Our inner ape: a leading primatologist explains why we are who we are, Nueva York, Riverhead Books.

Enloe, C (2001): “Closing Remarks”, International Peacekeeping, 8(2), pp. 111-113.

Fraser, N. (1997): Iustitia Interrupta. Reflexiones críticas desde la posición postsocialista, Bogotá, Siglo del Hombre-Universidad de Los Andes.

Gorlier, J. C. (2002): Comunidades Narrativas. El impacto de la praxis feminista sobre la teoría social, Argentina, Ediciones Al Margen.

Gutiérrez, Francisco (2015): “Una historia simple?”, Informe de la Comisión Histórica del Conflicto y sus Victimas (498-540). Mesa de Conversaciones. Disponible en: https://www.mesadeconversaciones.com.co/comunicados/informe-comisio $\% \mathrm{CC} \% 81 \mathrm{n}$ histo $\%$ CC $\% 81$ rica-del-conflicto-y-sus-vi $\%$ CC $\% 81$ ctimas-la-habana-febrero-de-2015 [Consulta: 28 de marzo de 2018]

Herrera M. C. y C. Pertuz (2015): "Narrativas femeninas del conflicto armado y la violencia política en Colombia: contar para rehacerse", Revista De Estudios Sociales, 53, pp. 150-162.

Higonnet, M. R. et al. (eds.) (1987): Behind the lines. Gender and the two World Wars, Londres, Yale University Press. 
Laclau, E. y C. Mouffe (1987): Hegemonía y estrategia socialista. Hacia una radicalización de la democracia, Madrid, Siglo XXI.

Lamus, D. (2010): De la subversión a la inclusión: movimiento de mujeres de la segunda ola en Colombia, 1975-200, Bogotá, Instituto Colombiano de Antropología e Historia.

Londoño, L. M. y Y. Fernanda Nieto (2006): Mujeres no contadas. Procesos de desmovilización y retorno a la vida civil de mujeres excombatientes en Colombia 1990-2003, Medellín, La carretera editores.

Magallón, C. (1998): "Sostener la vida, producir la muerte: estereotipos de género y violencia", en Vicent Fisas (ed.), El sexo de la violencia. Género y cultura de la violencia, Barcelona, Icaria.

Malesevic, S. (2010): The sociology of war and violence, UK, Cambridge University Press.

Mann, M. (1986): The sources of social power I: a history of power from the beginning to $A D$ 1760, UK, Cambridge University Press.

Marín, I. (2016): "Postconflicto en Colombia (4). Sin previa invitación: mujeres en la Habana", en Open Democracy 3/02/2016. Disponible en web:

https://www.opendemocracy.net/democraciaabierta/isabela-mar-n-carvajal/

postconflicto-en-colombia-4-sin-previa-invitaci-n-mujeres-e

[Consulta: 15 de marzo de 2018]

Meertens, D. (1995): “Mujer y violencia en los conflictos rurales”, Análisis Político, 24, pp. 36-49.

Mesa de Conversaciones (agosto 2016): Acuerdo Final para la terminación del conflicto y la construcción de una paz estable y duradera, entre el gobierno nacional y las Fuerzas Armadas Revolucionarias de Colombia-Ejército Popular (FARC-EP).

Mesa, M. (2012): "Prevención de conflictos, paz y seguridad: Avances conceptuales, experiencias y lecciones aprendidas", Pensamiento Propio, 36/37, pp. 189-219.

Millet, K. (1970): Política sexual, Madrid, Aguilar.

Moore, H. L. (1996): Antropología y feminismo, Madrid, Cátedra.

Murillo Sencail, X. (2004): Factibilidad de la política pública de desmovilización y reencuentro desde una perspectiva de género. Estudio de caso: partido Comunista de Colombia Marxista Leninista y Ejército Popular de Liberación, Tesis maestría inédita, Universidad Nacional de Colombia, Bogotá.

ONU Mujeres (2011): Informe Anual 2010-2011.

Osborne, R. (2009): Apuntes sobre violencia de género, Barcelona, Edicions Bellaterra.

Oxfam (2010): Violencia sexual en contra de las mujeres en el contexto de conflicto armado colombiano. Colombia 2001-2009, Colombia, Oxfam.

Pabón, R. (1984): Así nos tomamos la embajada, Bogotá, editorial Planeta.

PNH (1994): Programa de Reinserción, El programa de reinserción en Colombia: 1990-1994, Balance de un proceso, Santafé de Bogotá, Editorial Presidencia.

PNUD (2003): "El conflicto, callejón con salida. Informe Nacional de Desarrollo Humano para Colombia", Programa de Naciones Unidas para el Desarrollo, Bogotá. Disponible en: http://www.acnur.org/biblioteca/pdf/5626.pdf?view=1 [Consulta: 26 de abril de 2018]

Ramírez, C. (2015): “¿Qué palabra te dijera que llegue a tu corazón? Mujeres y proceso de negociación en Colombia", En Otras Palabras, 23, pp. 68-78.

Registro Único de Víctimas (RUV) Red Nacional de Información: https://rni.unidadvictimas.gov.co/RUV 
Resolución 1325/2000, Consejo de Seguridad, 31 de octubre de 2000, U.N. Doc. S/RED/1325 (2000).

Ruta Pacífica de las Mujeres (2013): La verdad de las mujeres. Víctimas del conflicto armado en Colombia, Colombia, Ruta Pacífica de las Mujeres.

Sader, E. e I. Jinkings (2009): Latinoamericana. Enciclopedia contemporánea de América latina y el Caribe, Madrid, Akal.

Said, E. (2002): Orientalismo, Madrid, Debate.

Salamanca, M. (2008): "Un ajedrez del conflicto armado colombiano", en Felipe Gómez (dir.), Colombia en su laberinto. Una mirada al conflicto, Madrid, La Catarata.

Sánchez, O. A. (2006): Nuevas formas de resistencia civil de lo privado a lo público. Movilizaciones de la Ruta Pacifica 1996-2003, Bogotá, Ruta Pacífica de las Mujeres.

Sánchez, O. et al. (2017): Encuesta de prevalencia de violencia sexual en contra de mujeres las mujeres en el contexto del conflicto armado colombiano (2010-2015), Bogotá, Campaña "Violaciones y otras violencias. Saquen mi cuerpo de la guerra".

Sánchez, M. L. y L. Rodríguez (2015): "Acciones colectivas de las organizaciones de mujeres por la paz en Colombia", Revista de Paz y Conflictos, 8 (2), pp. 149-177.

Service, E. R. (1978): Profiles in Ethnology, New York, Haper and Row.

Spivak, G. C. (1998): “PPuede hablar el sujeto subalterno?”, Orbis Tertius, 3 (6), pp.175-235. Disponible en:

http://www.memoria.fahce.unlp.edu.ar/art_revistas/pr.2732/pr.2732.pdf

[Consulta: 17 de mayo de 2017]

Squires, J. (2000): Gender in Political Theory, London, Wiley-Blackwell.

Tilly, C. (1985): "War Making and State Making as Organized Crime", en: P. Evans, D. Rueschemeyer and T. Skocpol (eds.), Bringing the State Back In, New York, Cambridge University Press.

Turriago, G. y J. M. Bustamante (2003): Estudio de los procesos de reinserción en Colombia 1991-1998, Bogotá, FIP.

Uprimny, R. (2014): “Una historia de Hombres", noticia en El Espectador, 06/07/2014. Disponible en:

http://www.elespectador.com/opinion/una-historia-de-hombres-columna-515137

[Consulta: 19 de marzo de 2018]

Uricoechea, F. (2000): "Perspectiva institucional de los procesos de reinserción", en Ministerio del Interior, De las armas a la democracia, tomo II, Bogotá, Ministerio del Interior.

Vásquez, M. E. (2001): "Entre la guerra y la paz: resignificación de los procesos de reinserción", en Ministerio del Interior, Instituto Luis Carlos Galán, De las armas a la democracia, Tomo II, Bogotá, Ministerio del Interior, 2000.

Velásquez, M. (2009): "El papel de las mujeres en los procesos de construcción de paz", en ¿Justicia Desigual? Género y Derechos de las víctimas en Colombia, Fondo de Desarrollo de las Naciones Unidas para la Mujer (UNIFEM), Bogotá, Colombia.

Weber, M. (1964): Economía y Sociedad, Madrid, Fondo de cultura económica.

White, M. (1992): "Deconstruction and therapy", en David Epston and Michael White, Experience, contradiction, narrative and imagination, Adelaide, Dulwich Center Publications.

Wills, M. E. (2007): “¿Inclusión sin representación? La irrupción política de las mujeres en Colombia”, La manzana de la discordia, (4), pp. 117-122. 
Wills, M. E. (2015): "Los tres nudos de la guerra colombiana: un campesinado sin representación política, una polarización social en el marco de una institucionalidad fracturada y unas articulaciones perversas entre regiones y centro", Informe de la Comisión Histórica del Conflicto y sus Víctimas (762-806), Mesa de Conversaciones, Disponible en:

https://www.mesadeconversaciones.com.co/comunicados/informe-comisio $\% \mathrm{CC} \% 81 \mathrm{n}$ histo $\% \mathrm{CC} \% 81$ rica-del-conflicto-y-sus-vi $\%$ CC $\% 81$ ctimas-la-habana-febrero-de-2015 [Consulta: 28 de marzo de 2018] 